\title{
DE
}

BULGARIAN ACADEMY OF SCIENCES

CYBERNETICS AND INFORMATION TECHNOLOGIES • Volume 14, No 3

Sofia • 2014

\section{Software Reliability Estimate with Duplicated Components Based on Connection Structure}

\author{
Zhen Li, Junfeng Tian, Pengyuan Zhao \\ College of Mathematics and Computer, Hebei University, Baoding 071002, Hebei, China \\ Emails: lizhen_hbu@126.com tjf@hbu.edu.cn zhaopengyuan@cmc.hbu.cn
}

\begin{abstract}
Reliability testing of complex software at the system level is impossible due to the environmental constraint or the time limitation, so its reliability estimate is often obtained based on the reliability of subsystems or components. The connection structure was defined and the component-based software reliability was estimated based on it. For the present popular software with duplicated components, an approach to variance estimation of software reliability for complex structure systems was proposed, which has improved the hierarchical decomposition approach of variance estimation just for series-parallel systems. Experimental results indicated that the approach to variance estimation for reliability of software with duplicated components has advantages, such as the simple calculation process, small error result, and suitability for complex structure systems. Finally, the sensitivity analysis, used to identify critical components for resource allocation, could better improve the software reliability.
\end{abstract}

Keywords: Component-based software, reliability, duplicated components, variance estimation, complex structure.

\section{Introduction}

For complex software, reliability testing at the system level is impossible due to the environmental constraint or the time limitation. In these situations, reliability can be estimated by conducting testing at subsystem or component levels. The software reliability estimate possesses uncertainty. In practice, people usually would rather select the software with lower and more accuracy reliability estimate than software with higher and less accuracy reliability estimate. 
A common practice in software design is to use the same components in different locations of the software due to the requirement of the same or similar functions. For the same components, their reliability is often inferred from the same testing sample, that is, the reliability estimates of the same component are $s$-dependent. For software with duplicated components, the variance of the software reliability estimate needs computation of higher order moments involving iterations and discrete convolutions. These procedures are often time-consuming. If we ignore the dependence of the component reliability estimates, the variance of the software reliability estimate will be underestimated $[1,2]$.

In recent years, researches on component-based software reliability have been paid more and more attention to. Mo ha me d, Z ul kernine [3] have proposed a simple CFG structure that represents inter-component and intra-component control flow transitions; $\mathrm{W}$ a $\mathrm{n} \mathrm{g}$ et al. [4] proposed a software reliability model which can deal with the cases of component interaction; F i o n d e l l a et al. [5] presented an efficient, scalable approach to analyze the reliability of a component-based software system considering the correlated component failures. These researches can estimate the component-based software reliability, but do not consider how to estimate its uncertainty.

For the uncertainty measurement of software reliability estimates, C o i t [6] demonstrated a flexible procedure to determine the confidence intervals for seriesparallel system reliability, when there was uncertainty regarding the component reliability information; Jin expanded Coit's method to estimate the confidence intervals for series-parallel systems with arbitrarily repeated components [1], and proposed a hierarchical decomposition procedure to determine the variance of the reliability estimate for series-parallel systems [2]. $\mathrm{H}$ i l l et al. [7] presented probability inequalities and results useful in defining the inequality-based reliability estimate for series-parallel system with repeated components. The above approaches are just for series-parallel systems and inadequate for complex structure systems.

On the basis of the above problems, the connection structure is defined and the component-based software reliability is estimated based on it. For software with duplicated components, an approach to variance estimation of software reliability for complex structure systems is proposed, which improves the hierarchical decomposition approach of variance estimation just for series-parallel systems proposed by J in [2].

\section{A connection structure for component-based software}

First of all, the component-based software is hierarchized to multi layers and each layer involves several modules, that is, the software is devised into multiple modules in a hierarchical order from the system level down to the component level. The rule to define a module is in two parts: 1) at least one component is included; and 2) within a module, there is only one connection structure for sub-modules or components. The connection structure is defined as follows:

Definition 1. A connection structure $A$ is defined as the connection structure of modules or components in the same layer for the hierarchical component-based 
software, denoted by $\mathrm{A}=\langle\mathrm{S}, \mathrm{P}, \mathrm{F}, \mathrm{B}, \mathrm{L}, \mathrm{SC}, \mathrm{BC}, \mathrm{LC}\rangle$ where the elements represent a Sequence structure, Parallel structure, Fault tolerance structure, Branch structure, Loop structure, Sequence Call structure, Branch Call structure and Loop Call structure respectively. If a module contains only one component, the default connection structure is $\mathrm{S}$, assuming that the component connects to itself.

Definition 2. A connection structure flowchart is the running flow chart of the hierarchical component-based software which expresses the connection structure of modules or components in the same layer.

Definition 3. The component-based software CS is denoted by $\mathrm{CS}=\{C(i), M(i), A(i) \mid i=1,2, \ldots, m\}$ where $m$ is the number of layers, $C(i)$ is the set of the $i$-th layer components, $M(i)$ is the set of the $i$-th layer modules, $A(i)$ is the set of connection structures of the $i$-th layer modules or components.

We assume that the transfer of control among components is a Markov process. Let the reliability of components $c_{i} i=1,2, \ldots, n$, be $r_{i}$ and the transition probability from $c_{i}$ to $c_{j}$ be $p_{i, j}, i, j=1,2, \ldots, n, p_{i, j} \in[0,1]$.

\subsection{Sequence structure}

Components of the sequence structure are executed in sequence. The component sequence reliability is the product of component reliability. The sequence's probability of occurrence is the product of each transition probability. Then the reliability of the sequence structure can be expressed as the product of component sequence reliability and the sequence's probability of occurrence. In Fig. 1(a), the reliability of a sequence structure $R_{\mathrm{S}}$ is

$$
R_{\mathrm{S}}=p_{1,2} p_{2,3} \ldots p_{n-1, n} r_{1} r_{2} \ldots r_{n}=\prod_{i=1}^{n-1} p_{i, i+1} \times \prod_{j=1}^{n} r_{j}
$$

\subsection{Parallel structure}

In concurrent environment, the performance of the system can be improved by running multiple components concurrently. The reliability of a parallel structure can be expressed as the product of component reliability. In Fig. 1b, the reliability of the parallel structure $R_{\mathrm{P}}$ is

$$
R_{\mathrm{P}}=r_{1} r_{2} \ldots r_{n}=\prod_{i=1}^{n} r_{i}
$$

\subsection{Fault tolerance structure}

Only one of the components with a fault tolerance structure runs at a certain time. In Fig. 1c, $c_{1} c_{2}, \ldots, c_{n}$, denoted by a dashed line, are the backup components of the primary component $c_{1}$. When all components in Fig. 1c fail, the fault tolerance structure is unreliable. The reliability of fault tolerance structure showed in Fig. 1c $R_{\mathrm{F}}$ is

$$
R_{\mathrm{F}}=1-\left(1-r_{1}\right)\left(1-r_{2}\right) \ldots\left(1-r_{n}\right)=1-\prod_{i=1}^{n}\left(1-r_{i}\right) .
$$




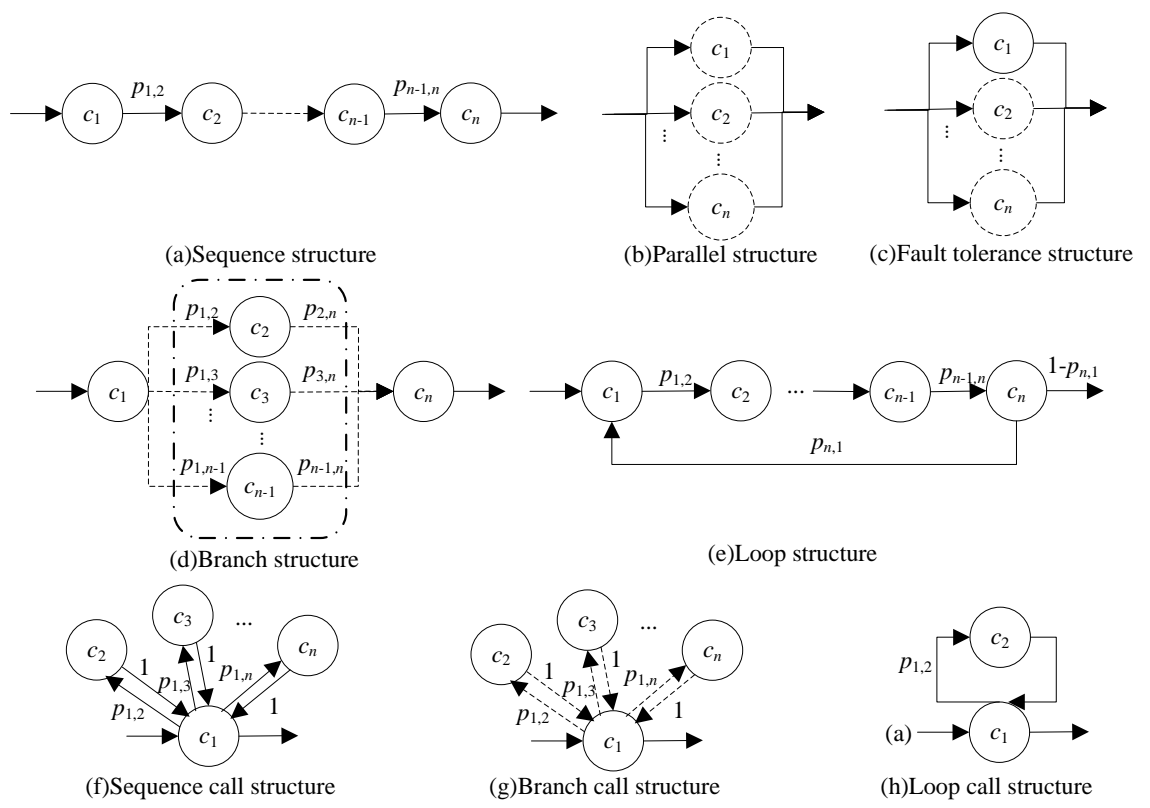

Fig. 1. A connection structure

\subsection{Branch structure}

In the branch structure, only one branch runs at a certain time. In Fig. 1d, the branch structure is denoted by a dash-dotted line. The reliability of the branch structure can be expressed as the sum of the product of branch reliability and the branch probability of occurrence. The reliability of the branch structure $R_{\mathrm{B}}$ is

$$
R_{\mathrm{B}}=p_{1,2} p_{2, n} r_{2}+p_{1,3} p_{3, n} r_{3}+\ldots+p_{1, n-1} p_{n-1, n} r_{n-1}=\sum_{i=2}^{n-1} p_{1, i} p_{i, n} r_{i} .
$$

\subsection{Loop structure}

Components with a loop structure are executed circularly. We add an entry component before the loop structure and an exit component after the loop structure in Fig. 1e. The reliability of the entry component $r_{\text {entry }}$ and the exit component $r_{\text {exit }}$ is 1 . Then the reliable state $R$ and the unreliable state $U$ are added as terminal states, representing the state of the reliable and unreliable output respectively, shown in Fig. 2; $p_{i, j} r_{i}$ represent the probability that the execution of $c_{i}$ produces the correct result and the control is transferred to $c_{j}$. We can gain the transition probability matrix $\boldsymbol{Q} ; \boldsymbol{Q}^{k}(1, n+2)$, the element of row 1 , column $n+2$ for the $k$-th power of matrix $\boldsymbol{Q}$ represents the probability that starting from an entry component, the chain enters the absorbing state $\{R, U\}$ at or before the $k$-th step. The value range of $k$ is $[0, \infty)$. If there is at least one component $c_{1}$ then $\boldsymbol{Q}^{0}(1, n+2)=\boldsymbol{Q}^{1}(1, n+2)=0$. Let $\boldsymbol{M}=\sum_{k=0}^{\infty} \boldsymbol{Q}^{k}=(\boldsymbol{I}-\boldsymbol{Q})^{-1}, \boldsymbol{M}(1, n+2)$, the element of row 1 , column $n+2$ for matrix 
$\boldsymbol{M}$, is the probability from an entry to an exit component. The reliability of the loop structure in Fig. 1e $R_{\mathrm{L}}$ is

$$
\begin{aligned}
& R_{\mathrm{L}}=\boldsymbol{M}(1, n+2) r_{\text {exit }}= \\
& \left\{\left[\left(1-p_{1,1}\right) r_{1}\right] /\left[1-p_{1,1} r_{1}\right], \quad n=1 ;\right. \\
& {\left[\left(1-p_{n, 1}\right) \times \prod_{i=1}^{n-1} p_{i, i+1} \times \prod_{j=1}^{n} r_{j}\right] /\left[1-p_{n, 1} \times \prod_{i=1}^{n-1} p_{i, i+1} \times \prod_{j=1}^{n} r_{j}\right], \quad n>1 .}
\end{aligned}
$$

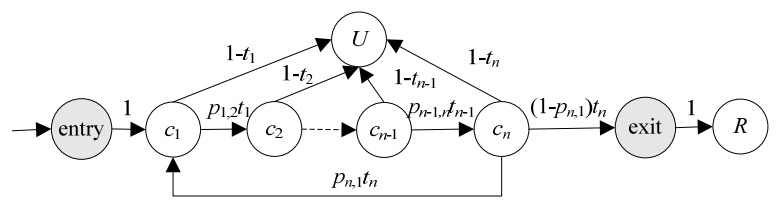

Fig. 2. A loop structure after adding an entry, exit and states

\subsection{Call structure}

There are dependent relations among the components with a call structure. We adopt the Continuation Passing Style (CPS) to transform these dependent relations. CPS transformation makes the caller component divided into several program segments, as sub-components, each of them does not calling other components. There are three types of call structures.

Type 1. Sequence call structure. The dependency relation in a sequence call structure is a sequence. In Fig. 1f, $c_{1}$ calls $c_{2}, \ldots, c_{n}$ in the sequence and the transition probability from $c_{2}$, or $c_{3}, \ldots$,or $c_{n}$ to $c_{1}$ is $1 . c_{1}$ is divided into subcomponents $c_{1,1}, c_{1,2}, \ldots, c_{1, n}$. The input of $c_{2}$ is the output of $c_{1,1}$, the input of $c_{1,2}$ is the output of $c_{2}, \ldots$, as shown in Fig. 3a. According to (1), the reliability of the sequence call structure $R_{\mathrm{SC}}$ is

$$
R_{\mathrm{SC}}=p_{1,2} p_{1,3} \ldots p_{1, n} r_{1,1} r_{1,2} \ldots r_{1, n} r_{2} \ldots r_{n}=\prod_{i=2}^{n} p_{1, i} \times \prod_{j=1}^{n} r_{1, j} \times \prod_{k=2}^{n} r_{k},,
$$

where $r_{1,1}, r_{1,2}, \ldots, r_{1, n}$ is the reliability of $c_{1,1}, c_{1,2}, \ldots, c_{1, n}$ respectively.

Type 2. Branch call structure. The dependency relation in a branch call structure is a branch. In Fig. $1 \mathrm{~g} c_{1}$ calls $c_{2}$, or $c_{3}, \ldots$, or $c_{n}$ and the transition probability from $c_{2}, c_{3}, \ldots, c_{n}$ to $c_{1}$ is $1 . c_{1}$ is divided into $c_{1,1}$ and $c_{1,2}$. In Fig. 3b, $c_{1,1}$ is the part of $c_{1}$ before the branch and $c_{1,2}$ is the part of $c_{1}$ after the branch. The reliability of the branch structure can be calculated by (4). The reliability of the branch call structure $R_{\mathrm{BC}}$ is

$$
R_{\mathrm{BC}}=r_{1,1} r_{1,2}\left(p_{1,2} r_{2}+p_{1,3} r_{3}+\ldots+p_{1, n} r_{n}\right)=r_{1,1} r_{1,2} \sum_{i=2}^{n} p_{1, i} r_{i} .
$$

Type 3. Loop call structure. The dependency relation in a loop call structure is a loop. In Fig. 1h, $c_{1}$ calls $c_{2}$ circularly and the transition probability from $c_{2}$ to 
$c_{1}$ is 1. $c_{1}$ is divided into $c_{1,1}$ and $c_{1,2}$. In Fig. 3c, $c_{1,1}$ is the part of $c_{1}$ before calling $c_{2}$ circularly, and $c_{1,2}$ is the part of $c_{1}$ after calling $c_{2}$ circularly. The reliability of the loop structure can be calculated by (5). The reliability of the loop call structure $R_{\mathrm{LC}}$ is

(8) $R_{\mathrm{LC}}=r_{1,1} r_{1,2} p_{1,2} \times\left[\left(1-p_{1,2}\right) r_{2}\right] /\left(1-p_{2,1} r_{2}\right)=\left[p_{1,2}\left(1-p_{1,2}\right) r_{1,1} r_{1,2} r_{2}\right] /\left(1-p_{2,1} r_{2}\right)$.

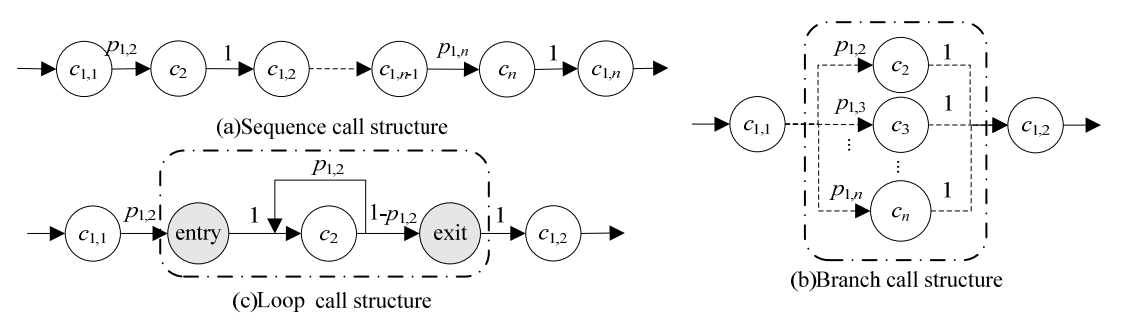

Fig. 3. A call structure after division

\section{Reliability estimate of component-based software}

It is easily proved that any component-based software can be expressed by a connection structure flowchart. Without loss of generality, the connection structure flowchart of component-based software in Fig. 4a is used as an example. The main modules are denoted by a dash-dotted line in each layer. The layer 0 of software is denoted by $S_{0} ; S_{u, v} u, v=1,2, \ldots$, indicating that the sub-modules or components are connected in a sequence at its lower adjacent layer and it is the $v$-th module in layer $u$. The first subscript indicates the layer number, while the second subscript indicates the module number on that layer. Modules with other connection structures can be indicated by a similar expression. The hierarchical model of component-based software is shown in Fig. 4b.

To standardize the hierarchy, artificial modules, denoted by a dashed line, are introduced to the intermediate layers, such that all modules at one layer can only communicate with their adjacent layers. The overall software reliability $R$ in Fig. 4a can be expressed recursively as

$$
\begin{aligned}
R= & R_{S_{0}}\left(R_{S_{1,1}}\left[R_{S_{2,1}}\left(r_{1}\right)\right], R_{L_{1,2}}\left[R_{L_{2,2}}\left(r_{2}, r_{3}\right)\right], R_{F_{1,3}}\left[R_{F_{2,3}}\left(r_{4}, r_{5}\right)\right],\right. \\
& \left.R_{B_{1,4}}\left[R_{S_{2,4}}\left(r_{1}, r_{6}\right), R_{S C_{2,5}}\left(r_{7}, r_{1}\right)\right], R_{S_{1,5}}\left[R_{S_{2,6}}\left(r_{3}\right)\right]\right) .
\end{aligned}
$$

The true component reliability $r_{i}, i=1,2, \ldots, 7$, is usually not available, and the estimate $\hat{r}_{i}$ is often used to substitute $r_{i}$. For $c_{i}, n$ samples are tested for a period of time $t$ and the number of failures is $f$. The reliability of $c_{i}$ can be estimated using a binomial distribution [6]: $\hat{r}_{i}=(n-f) / n, \quad \operatorname{var}\left(\hat{r}_{i}\right)=\left[\hat{r}_{i}\left(1-\hat{r}_{i}\right)\right] / n$.

After substituting $\hat{r}_{i}$ into (9), the software reliability estimate becomes

$$
\begin{gathered}
\hat{R}=R_{S_{0}}\left(R_{S_{1,1}}\left[R_{S_{2,1}}\left(\hat{r}_{1}\right)\right], R_{L_{1,2}}\left[R_{L_{2,2}}\left(\hat{r}_{2}, \hat{r}_{3}\right)\right], R_{F_{1,3}}\left[R_{F_{2,3}}\left(\hat{r}_{4}, \hat{r}_{5}\right)\right],\right. \\
\left.R_{B_{1,4}}\left[R_{S_{2,4}}\left(\hat{r}_{1}, \hat{r}_{6}\right), R_{S C_{2,5}}\left(\hat{r}_{7}, \hat{r}_{1}\right)\right], R_{S_{1,5}}\left[R_{S_{2,6}}\left(\hat{r}_{3}\right)\right]\right) .
\end{gathered}
$$


If the mean of $\hat{r}_{i}$ (i.e., $\bar{r}_{i}=E\left[\hat{r}_{i}\right]$ ) is substituted into (9), the mean of the software reliability estimate can be approximated as

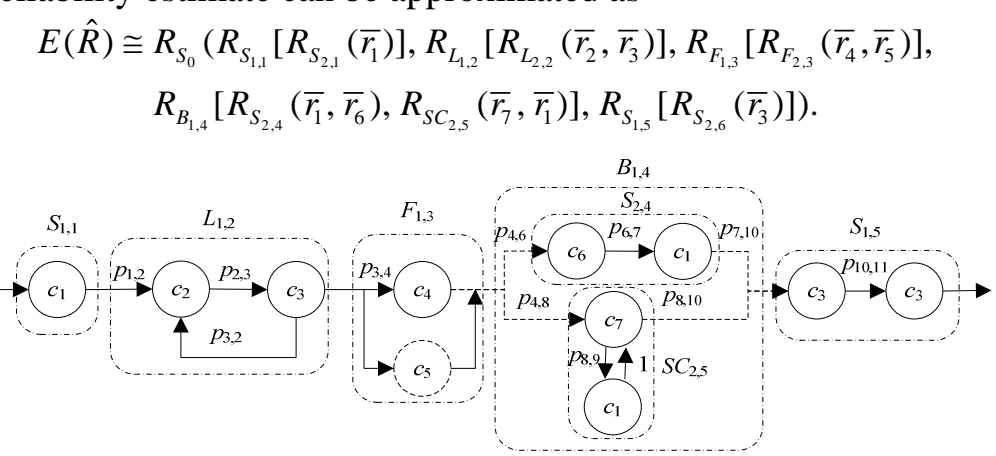

(a) Connection structure flowchart

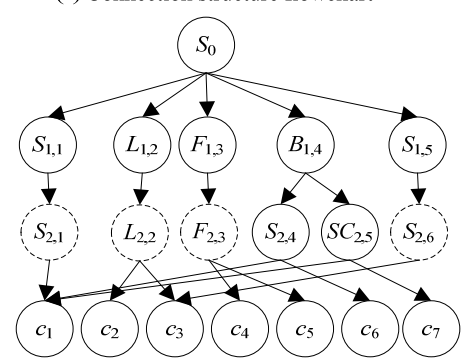

(b) The hierarchical model

Fig. 4. An example of component-based software

We assume that the uncertainties of the component reliability estimates are relatively small and the standard deviation, divided by the mean value, is less than 0.3 [8]. We expand (10) at the nominal mean $E(\hat{R})$, using the first-order Taylor series. We obtain

$$
\hat{R} \cong E(\hat{R})+\sum_{i=1}^{7} b_{i}\left(\hat{r}_{i}-\bar{r}_{i}\right),
$$

where

$$
\begin{gathered}
b_{1}=\frac{\partial R_{S_{0}}}{\partial R_{S_{1,1}}} \frac{\partial R_{S_{1,1}}}{\partial R_{S_{2,1}}} \frac{\partial R_{S_{2,1}}}{\partial r_{1}}+\frac{\partial R_{S_{0}}}{\partial R_{B_{1,4}}} \frac{\partial R_{B_{1,4}}}{\partial R_{S_{2,4}}} \frac{\partial R_{S_{2,4}}}{\partial r_{1}}+\frac{\partial R_{S_{0}}}{\partial R_{B_{1,4}}} \frac{\partial R_{B_{1,4}}}{\partial R_{S C_{2,5}}} \frac{\partial R_{S_{2,5}}}{\partial r_{1}}, \\
b_{2}=\frac{\partial R_{S_{0}}}{\partial R_{L_{1,2}}} \frac{\partial R_{L_{1,2}}}{\partial R_{L_{2,2}}} \frac{\partial R_{L_{2,2}}}{\partial r_{2}}, b_{3}=\frac{\partial R_{S_{0}}}{\partial R_{L_{1,2}}} \frac{\partial R_{L_{1,2}}}{\partial R_{L_{2,2}}} \frac{\partial R_{L_{2,2}}}{\partial r_{3}}+\frac{\partial R_{S_{0}}}{\partial R_{S_{1,5}}} \frac{\partial R_{S_{1,5}}}{\partial R_{S_{2,6}}} \frac{\partial R_{S_{2,6}}}{\partial r_{3}}, \\
b_{4}=\frac{\partial R_{S_{0}}}{\partial R_{F_{1,3}}} \frac{\partial R_{F_{1,3}}}{\partial R_{F_{2,3}}} \frac{\partial R_{F_{2,3}}}{\partial r_{4}}, b_{5}=\frac{\partial R_{S_{0}}}{\partial R_{F_{1,3}}} \frac{\partial R_{F_{1,3}}}{\partial R_{F_{2,3}}} \frac{\partial R_{F_{2,3}}}{\partial r_{5}}, b_{6}=\frac{\partial R_{S_{0}}}{\partial R_{B_{1,4}}} \frac{\partial R_{B_{1,4}}}{\partial R_{S_{2,4}}} \frac{\partial R_{S_{2,4}}}{\partial r_{6}}, \\
b_{7}=\frac{\partial R_{S_{0}}}{\partial R_{B_{1,4}}} \frac{\partial R_{B_{1,4}}}{\partial R_{S C_{2,5}}} \frac{\partial R_{S C_{2,5}}}{\partial r_{7}} .
\end{gathered}
$$


All derivatives are evaluated at the nominal value of the component reliability estimates. For example, $\left(\partial R_{S_{0}} / \partial R_{S_{1_{1}}}\right)=\left.\left(\partial R_{S_{0}} / \partial R_{S_{1,1}}\right)\right|_{\left(\bar{r}_{1}, \bar{r}_{2}, \bar{r}_{3}, \bar{T}_{4}, \bar{r}_{5}, \bar{r}_{6}, \bar{r}_{7}\right)}$. Because

$$
\begin{gathered}
R_{S_{0}}=p_{1,2} p_{3,4} R_{S_{1,1}} R_{L_{1,2}} R_{F_{1,3}} R_{B_{1,4}} R_{S_{1,5}}, R_{S_{1,1}}=R_{S_{2,1}}, R_{L_{1,2}}=R_{L_{2,2}}, R_{F_{1,3}}=R_{F_{2,3}}, \\
R_{B_{1,4}}=p_{4,6} p_{7,10} R_{S_{2,4}}+p_{4,8} p_{8,10} R_{S C_{2,5}}, R_{S_{1,5}}=R_{S_{2,6}}, R_{S_{2,1}}=r_{1}, \\
R_{L_{2,2}}=p_{2,3} r_{2} r_{3} /\left(1-p_{2,3} p_{3,2} r_{2} r_{3}\right), R_{F_{2,3}}=r_{4}+r_{5}-r_{4} r_{5}, R_{S_{2,4}}=p_{6,7} r_{1} r_{6}, R_{S C_{2,5}}=p_{8,9} r_{1} r_{7}, \\
R_{S_{2,6}}=p_{10,11} r_{1}^{2},
\end{gathered}
$$

the coefficient $b_{i}$ can be obtained as

$$
\begin{gathered}
b_{1}=p_{1,2} p_{3,4} \bar{R}_{L_{1,2}} \bar{R}_{F_{1,3}} \bar{R}_{B_{1,4}} \bar{R}_{S_{1,5}}+p_{1,2} p_{3,4} p_{4,6} p_{6,7} p_{8,9} p_{7,10} \bar{R}_{S_{1,1}} \bar{R}_{L_{1,2}} \bar{R}_{F_{1,3}} \bar{R}_{\mathrm{S}_{1,5}} \bar{r}_{6}+ \\
p_{1,2} p_{3,4} p_{4,8} p_{8,10} \bar{R}_{S_{1,1}} \bar{R}_{L_{1,2}} \bar{R}_{F_{1,3}} \bar{R}_{S_{1,5}} \bar{r}_{7}, b_{2}=p_{1,2} p_{2,3} p_{3,4} \bar{R}_{S_{1,4}} \bar{R}_{F_{1,3}} \bar{R}_{\mathrm{B}_{1,4}} \bar{R}_{S_{1,5}} \bar{r}_{3} /\left(1-p_{2,3} p_{3,2} \bar{r}_{2} \bar{r}_{3}\right)^{2}, \\
b_{3}=p_{1,2} p_{2,3} p_{3,4} \bar{R}_{S_{1,1}} \bar{R}_{F_{1,3}} \bar{R}_{B_{1,4}} \bar{R}_{S_{1,5}} \bar{r}_{2} /\left(1-p_{2,3} p_{3,2} \bar{r}_{2} \bar{r}_{3}\right)^{2}+2 p_{1,2} p_{3,4} p_{9,10} \bar{R}_{S_{1,1}} \bar{R}_{L_{1,2}} \bar{R}_{F_{1,3}} \bar{R}_{B_{1,4}} \bar{r}_{1}, \\
b_{4}=p_{1,2} p_{3,4} \bar{R}_{S_{1,1}} \bar{R}_{L_{1,2}} \bar{R}_{B_{1,4}} \bar{R}_{S_{1,5}}\left(1-\bar{r}_{5}\right), b_{5}=p_{1,2} p_{3,4} \bar{R}_{S_{1,1}} \bar{R}_{L_{1,2}} \bar{R}_{B_{1,4}} \bar{R}_{S_{1,5}}\left(1-\bar{r}_{4}\right), \\
b_{6}=p_{1,2} p_{3,4} p_{4,6} p_{6,7} p_{7,9} \bar{R}_{S_{1,1}} \bar{R}_{L_{1,2}} \bar{R}_{F_{1,3}} \bar{R}_{S_{1,5}} \bar{r}_{1}, b_{7}=p_{1,2} p_{3,4} p_{4,8} p_{8,9} p_{8,10} \bar{R}_{S_{1,1}} \bar{R}_{L_{1,2}} \bar{R}_{F_{1,3}} \bar{R}_{\mathrm{S}_{1,5}} \bar{r}_{1} .
\end{gathered}
$$

We rearrange (12) and obtain

$$
(\hat{R}-E(\hat{R}))^{2} \cong \sum_{i=1}^{7} b_{i}^{2}\left(\hat{r}_{i}-\bar{r}_{i}\right)^{2}+\sum_{i \neq j}^{7} b_{i} b_{j}\left(\hat{r}_{i}-\bar{r}_{i}\right)\left(\hat{r}_{j}-\bar{r}_{j}\right) .
$$

Taking the expectation of both sides of (13), the variance of the software reliability estimate is

$$
\operatorname{vâr}(\hat{R}) \cong \sum_{i=1}^{7} b_{i}^{2} E\left[\left(\hat{r}_{i}-\bar{r}_{i}\right)^{2}\right]=\sum_{i=1}^{7} b_{i}^{2} \operatorname{vâr}\left(\hat{r}_{i}\right) .
$$

We notice that only $\bar{r}_{i}$ and $\operatorname{var}\left(\hat{r}_{i}\right)$ are needed for estimating $b_{i}$ and $\operatorname{vâr}(\hat{R})$. Therefore, our model does not require computation of the higher order moments of the component reliability estimates. Hence, it significantly simplifies the computation steps of $\operatorname{vâr}(\hat{R})$.

\section{Experiments and analyses}

Our experiment has been carried out by using an ATM bank system [9]. The software system structure is shown in Fig. 5a. It consists of ten components and components $c_{2}, c_{3}, c_{5}, c_{6}$ and $c_{9}$ contains a natural fault respectively. Fig. $5 \mathrm{~b}$ shows the connection structure flowchart of it.

4.1. Software reliability estimate and comparison of the approaches to variance estimation

According to these five faults, five versions of the software were constructed and each version contained one fault. We randomly generated inputs to estimate the reliability of each individual faulty component until it was converged. The 
operational behaviours were collected to calculate the transition probability. $c_{5}$ is divided into sub-components $c_{5,1}$ and $c_{5,2}$. The reliability estimates are as follows: $\hat{r}_{1}=1.000, \quad \hat{r}_{2}=0.987, \quad \hat{r}_{3}=0.998, \quad \hat{r}_{4}=1.000, \quad \hat{r}_{5,1}=1.000, \quad \hat{r}_{5,2}=0.996, \quad \hat{r}_{6}=0.994$, $\hat{r}_{7}=1.000, \hat{r}_{8}=1.000, \hat{r}_{9}=0.976, \hat{r}_{10}=1.000$. The transition probabilities are shown in Fig. 5b.

For the hierarchical model of ATM bank system, the modules or components at each layer are shown in Table 1 . The software reliability estimate is 0.864 according to the approach in Section 3. Due to limitations in space, the detail calculation process is omitted.

The traditional approaches to variance estimation consider the dependence of the components, and need computation of higher order moments. These procedures are often time-consuming. If we assume that the components are s-independent, the estimation process will be facilitated, yet the variance of the software reliability estimate will be underestimated [1, 2]. Jin's hierarchical decomposition approach [2] does not need the computation of higher order moments, but it is just for seriesparallel systems. Our approach can be suitable for complex structure systems with duplicated components.

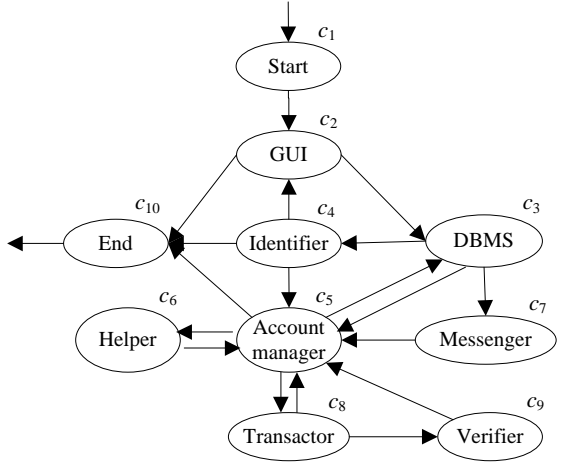

(a) System structure

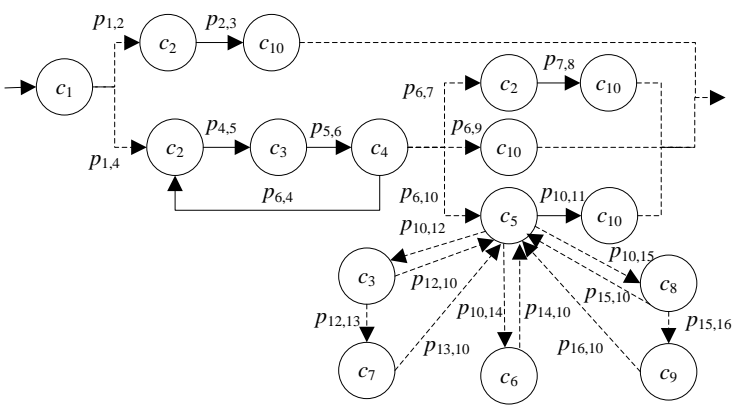

$p_{1,2}=0.001, p_{1,4}=0.999, p_{2,3}=1.000, p_{4,5}=1.000, p_{5,6}=1.000, p_{6,4}=0.047$ $p_{6,7}=0.001, p_{6,9}=0.001, p_{6,10}=0.951, p_{7,8}=1.000, p_{10,11}=1.000, p_{10,12}=0.436$, $p_{10,14}=0.100, p_{10,15}=0.464, p_{12,10}=0.781, p_{12,13}=0.219, p_{13,10}=1.000$, $p_{14,10}=1.000, p_{15,10}=0.010, p_{15,16}=0.990, p_{16,10}=1.000$.

$$
\text { (b) Connection structure flowchart }
$$

Fig. 5. ATM bank system structure and its connection structure flowchart 
Table 1 . The set of modules or components for each layer

\begin{tabular}{|c|c|}
\hline Layer & The set of modules or components \\
\hline 0 & $S_{0}=\left\{S_{1,1}, B_{1,2}\right\}$ \\
\hline 1 & $S_{1,1}=\left\{S_{2,1}\right\}, B_{1,2}=\left\{S_{2,2}, S_{2,3}\right\}$ \\
\hline 2 & $S_{2,1}=\left\{S_{3,1}\right\}, S_{2,2}=\left\{S_{3,2}\right\}, S_{2,3}=\left\{L_{3,3}, B_{3,4}\right\}$ \\
\hline 3 & $S_{3,1}=\left\{S_{4,1}\right\}, S_{3,2}=\left\{S_{4,2}\right\}, L_{3,3}=\left\{L_{4,3}\right\}, B_{3,4}=\left\{S_{4,4}, S_{4,5}, S_{4,6}\right\}$ \\
\hline 4 & $S_{4,1}=\left\{S_{5,1}\right\}, S_{4,2}=\left\{S_{5,2}\right\}, L_{4,3}=\left\{L_{5,3}\right\}, S_{4,4}=\left\{S_{5,4}\right\}, S_{4,5}=\left\{S_{5,5}\right\}, S_{4,6}=\left\{B C_{5,6}, S_{5,7}\right\}$ \\
\hline 5 & $\begin{array}{l}S_{5,1}=\left\{S_{6,1}\right\}, S_{5,2}=\left\{S_{6,2}\right\}, L_{5,3}=\left\{L_{6,3}\right\}, S_{5,4}=\left\{S_{6,4}\right\}, S_{5,5}=\left\{S_{6,5}\right\}, B C_{5,6}=\left\{S_{6,6}, B_{6,7}\right\} \\
S_{5,7}=\left\{S_{6,8}\right\}\end{array}$ \\
\hline 6 & $\begin{array}{l}S_{6,1}=\left\{S_{7,1}\right\}, S_{6,2}=\left\{S_{7,2}\right\}, L_{6,3}=\left\{L_{7,3}\right\}, S_{6,4}=\left\{S_{7,4}\right\}, S_{6,5}=\left\{S_{7,5}\right\}, S_{6,6}=\left\{S_{7,6}\right\}, B_{6,7}=\left\{B_{7,7},\right. \\
\left.S_{7,8}, B_{7,9}\right\}, S_{6,8}=\left\{S_{7,10}\right\}\end{array}$ \\
\hline 7 & $\begin{array}{l}S_{7,1}=\left\{S_{8,1}\right\}, S_{7,2}=\left\{S_{8,2}\right\}, L_{7,3}=\left\{L_{8,3}\right\}, S_{7,4}=\left\{S_{8,4}\right\}, S_{7,5}=\left\{S_{8,5}\right\}, S_{7,6}=\left\{S_{8,6}\right\}, B_{7,7}=\left\{S_{8,7},\right. \\
\left.S_{8,8}\right\}, S_{7,8}=\left\{S_{8,9}\right\}, B_{7,9}=\left\{S_{8,10}, S_{8,11}\right\}, S_{7,10}=\left\{S_{8,12}\right\}\end{array}$ \\
\hline 8 & $\begin{array}{l}S_{8,1}=\left\{c_{1}\right\}, S_{8,2}=\left\{c_{2}, c_{10}\right\}, L_{8,3}=\left\{c_{2}, c_{3}, c_{4}\right\}, S_{8,4}=\left\{c_{2}, c_{10}\right\}, S_{8,5}=\left\{c_{10}\right\}, S_{8,6}=\left\{c_{5}\right\}, \\
S_{8,7}=\left\{c_{3}\right\}, S_{8,8}=\left\{c_{3}, c_{7}\right\}, S_{8,9}=\left\{c_{6}\right\}, S_{8,10}=\left\{c_{8}\right\}, S_{8,11}=\left\{c_{8}, c_{9}\right\}, S_{8,12}=\left\{c_{10}\right\}\end{array}$ \\
\hline 9 & $\left\{c_{1}, c_{2}, c_{3}, c_{4}, c_{5}, c_{6}, c_{7}, c_{8}, c_{9}, c_{10}\right\}$ \\
\hline
\end{tabular}

\subsection{Sensitivity analysis}

Sensitivity analysis allows insight into the impact of changing the component reliability with respect to the software reliability. It can help to identify the critical components for a resource allocation. The critical point of software reliability $R$ with respect to a component reliability $r_{i}$ can be defined as $C_{R, i}=\Delta R / \Delta r_{i}$. The higher valued critical point indicates the critical component.

The critical points of changing the component reliability are shown in Fig. 6. Take the increment of a component reliability from 0.8 up to 0.85 for example, we suppose that the initial reliability of each component is 0.8 , and we start to increase each one from 0.8 to 0.85 in turn and observe its impact on the software reliability. We repeatedly change the different increments of each component and present the critical point of it. Fig. 6 shows that as the component reliability $r_{i}$ increases, the value of $C_{R, i}$ becomes high. The criticality of all components increases sharply, except $C_{R, 6}$ and $C_{R, 7}$ in a small increment. The critical values of the components $c_{3}, c_{5,1}$ and $c_{5,2}$ are higher than those of other components and the critical values of the components $c_{6}$ and $c_{7}$ are lower than those of the other components, which conforms to the actual application of an ATM bank system and is in accordance with the critical points of the software reliability with respect to the component reliabilities in [9]. The software reliability can be improved more efficiently if the critical components are reliable.

\section{Conclusions and future work}

For the present popular software with duplicated components, an approach to software reliability estimate and its variance estimation for complex structure systems is proposed, which has important applications in software reliability analysis with duplicated components. There is one condition in our approach, that 
is, the uncertainties of the component reliability estimates must be relatively small. This condition is often satisfied in a risk-averse software design environment or during the manufacturing processes where the parameters often shifted around the nominal ones within a small range. The future work will be focused on the extension of the decomposition method to general software or manufacturing processes, where the condition of small variations is violated.

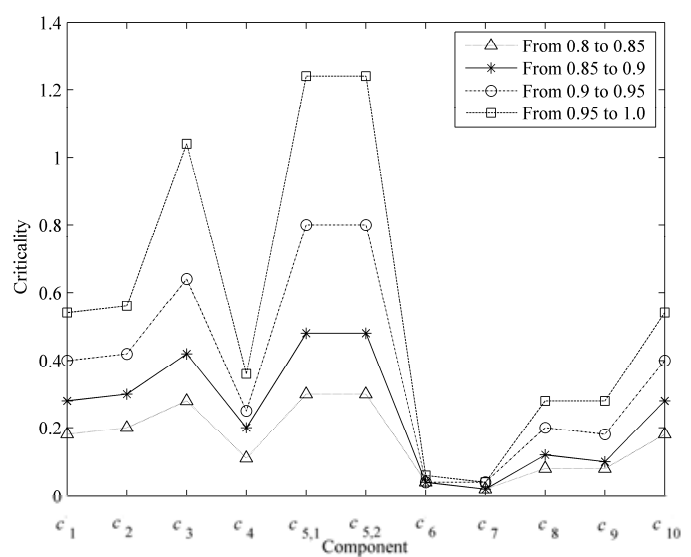

Fig. 6. The criticality of changing the component reliability

Acknowledgement: This paper was supported by the National Natural Science Foundation of China (61170254), Natural Science Foundation of Hebei Province (F2012201145), Science and Technology Research and Development Guidance Plan Project of Baoding City (13ZG012) and the Science Foundation of Hebei University(2013250).

\section{References}

1. Jin, T., D. C o it. Variance of System-Reliability Estimates with Arbitrarily Repeated Components. - IEEE Transactions on Reliability, Vol. 50, 2001, No 4, 409-413.

2. Ji n, T. Hierarchical Variance Decomposition of System Reliability Estimates with Duplicated Components. - IEEE Transactions on Reliability, Vol. 57, 2008, No 4, 564-573.

3. Mohamed, A., M. Zulkernine. A Control Flow Representation for Component-Based Software Reliability Analysis. - In: Proceedings of International Conference on Software Security and Reliability, IEEE, 2008, 1-10.

4. W a n g, Q., Y. L u, Z. X u, J. H a n. Software Reliability Model for Component Interaction Mode. - Journal of Electronics (China), Vol. 28, 2011, No 4, 632-642.

5. F i o n d e lla, L., S. Ra ja s e karan, S. S. G o kh a l e. Efficient Software Reliability Analysis with Correlated Component Failures. - IEEE Transactions on Reliability, Vol. 62, 2013, No 1, 244-255.

6. C o it, D. System-Reliability Confidence-Intervals for Complex-Systems with Estimated Component-Reliability. - IEEE Transactions on Reliability, Vol. 46, 1997, No 4, 487-493.

7. Hill, S. D., J. C. Spall, C. J. Maranzano. Inequality-Based Reliability Estimates for Complex Systems. - Naval Research Logistics, Vol. 60, 2013, No 5, 367-374.

8. C h e n, Y., B. L i, C. Y u e. Total Sensitivity Index Calculation via Error Propagation Equation. In: Proceedings of International Conference on Innovative Computing, IEEE, 2007, 619 p.

9. H su, C., C. Huang. An Adaptive Reliability Analysis Using Path Testing for Complex Component-Based Software Systems. - IEEE Transactions on Reliability, Vol. 60, 1997, No $1,158-170$. 